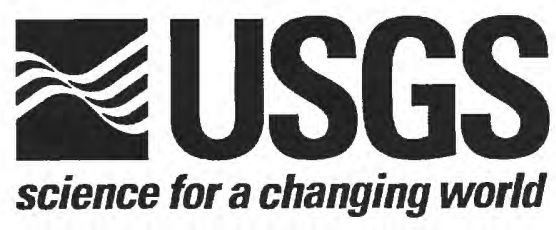

\title{
Geochemical and lead-isotopic studies of river sediment from major tributaries, upper Arkansas River watershed, Colorado
}

by

\author{
S.E. Church ${ }^{1}$, D.L. Fey ${ }^{1}$, and D.M. Unruh ${ }^{1}$
}

Open-File Report 00-337

2000

This report is preliminary and has not been reviewed for conformity with U.S. Geological Survey editorial standards and stratigraphic nomenclature. Any use of trade, product, or firm names is for descriptive purposes only and does not imply endorsement by the U.S. Government.

\section{U.S. DEPARTMENT OF THE INTERIOR}

U.S. GEOLOGICAL SURVEY

${ }^{1}$ Denver, Colorado 


\section{Geochemical and lead-isotopic studies of river sediment from major tributaries, upper Arkansas River watershed, Colorado}

The Arkansas River has its headwaters in the Rocky Mountains of central Colorado (fig. 1). This area has a long history of mineral resource extraction beginning in the 1860's with the discovery of placer gold at Leadville, Colo. Within the upper Arkansas River drainage basin, there are numerous mining districts (see fig. 1), some of which have had major mineral production. In addition to the mining activity, there were also a number of mills and smelters which processed the ores. Mining practices of the past have resulted in exposure of mine waste which, in turn, has resulted in accelerated weathering of pyrite and other sulfide minerals. The weathering process produces elevated concentrations of several metals in many of the stream reaches within the upper part of the drainage basin. The U.S. Geological Survey (USGS) is currently cooperating with other Federal agencies to conduct an environmental assessment of the upper Arkansas River basin using both element distribution maps and profiles, and lead-isotope fingerprinting to determine the potential extent of environmental degradation of the Arkansas River and its impact on riparian and aquatic habitat.

Phase I of this study was initiated in July, 1993 to examine the distribution of elements in river sediments from the Arkansas River basin in Lake County, Colo. (Church and others, 1993). The objective of that study. was to determine the origin and time-of-deposition of a fluvial tailings deposit in the Arkansas River immediately south of the confluence with California Gulch. We sampled the Arkansas River and its major tributaries (fig. 2, phase I sample sites) to evaluate the contribution of lead from each of the potential sources north of the fluvial tailings deposit. Cores of river sediments were taken at selected sites along the Arkansas River to provide sedimentological and geochronological control.

In phase II of the study, we retrieved geochemical data from numerous geologic studies conducted over the last several decades to prepare geochemical maps showing the distribution of copper, lead, and zinc in the upper Arkansas River basin (Smith, 1994). As a result of this work, we identified ten additional source areas in the Arkansas River basin where the concentration of lead in stream sediments exceeds 400 parts per million (ppm), seven areas where it exceeds 200 $\mathrm{ppm}$, and fourteen areas where it exceeds $100 \mathrm{ppm}$ in addition to the known source of metals in the Leadville mining district. These thresholds are respectively 30, 15, and 8 times that of crustal abundance of lead as defined in Fortescue (1992). Multi-element geochemical data from several thousand stream-sediment samples provided an excellent resource to guide our selection of sample sites along the Arkansas River. Potential source areas include numerous old mining districts and several milling and industrial sites. Using these geochemical maps, seventeen sample sites along the course of the Arkansas River and a composite core sample from Pueblo Reservoir were selected for geochemical and lead-isotopic analysis (fig. 2; phase II sample sites). Geologic, geomorphic, wildlife habitat, land access, and land ownership were also evaluated during the siteselection process.

In phase III of the study, we report results from analyses of the tributaries to the Arkansas River. Tributary streams to the Arkansas River were sampled to determine whether addiotonal sources of metal released from historical mining activities elsewhere in the Arkansas River watershed contribute to the metals in streambed sediment in the main stem of the 
Arkansas River. Whereas local anthropogenic sources were found in some of the tributaries, the measured chemical and lead-isotopic compositions determined at the mouths of these tributaries indicates that there are not substantial sources of metals from the tributaries that impact the streambed sediment in the Arkansas River.

Sampling methods and analytical procedures were reported in Church and others (1993; 1994). Sample localities are in fig. 2. Localities of NURE samples are in Smith (1997) and of two core samples (table 3, samples ARK-TYS1 and ARK-TYS13) from Lake County provided in Walton-Day and others $(1999$, p. 81$)$. Total digestion data are reported in table 1 , partial digestion data from the $2 \mathrm{M} \mathrm{HCl}-1$ percent $\mathrm{H}_{2} \mathrm{O}_{2}$ digestion are in table 2 , and the lead-isotopic data from analyses completed are in table 3. Results from this phase of the study have been requested in support of the Natural Resource Damage Assessment and Restoration report currently being prepared. The data are released here without interpretation and are not complete due to the fact that funding was not forthcoming to complete the study. Some samples analyzed are from the NURE samples collected in the 1970s (Smith, 2000). 


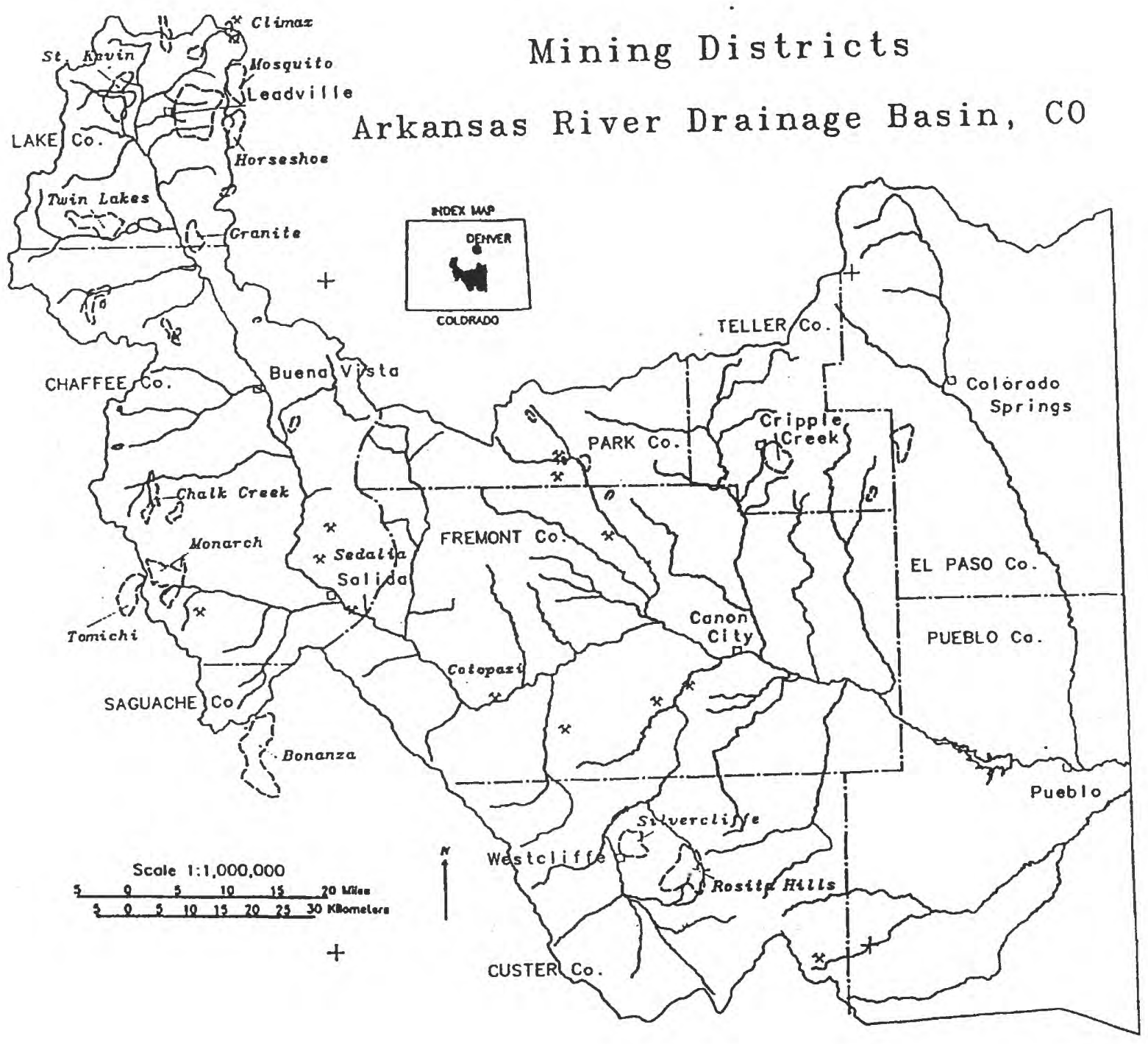

Figure 1. Map of the Arkansas River basin study area showing major gold and base-metal mining districts; some district boundaries are from Streufert and Davis (1990). 


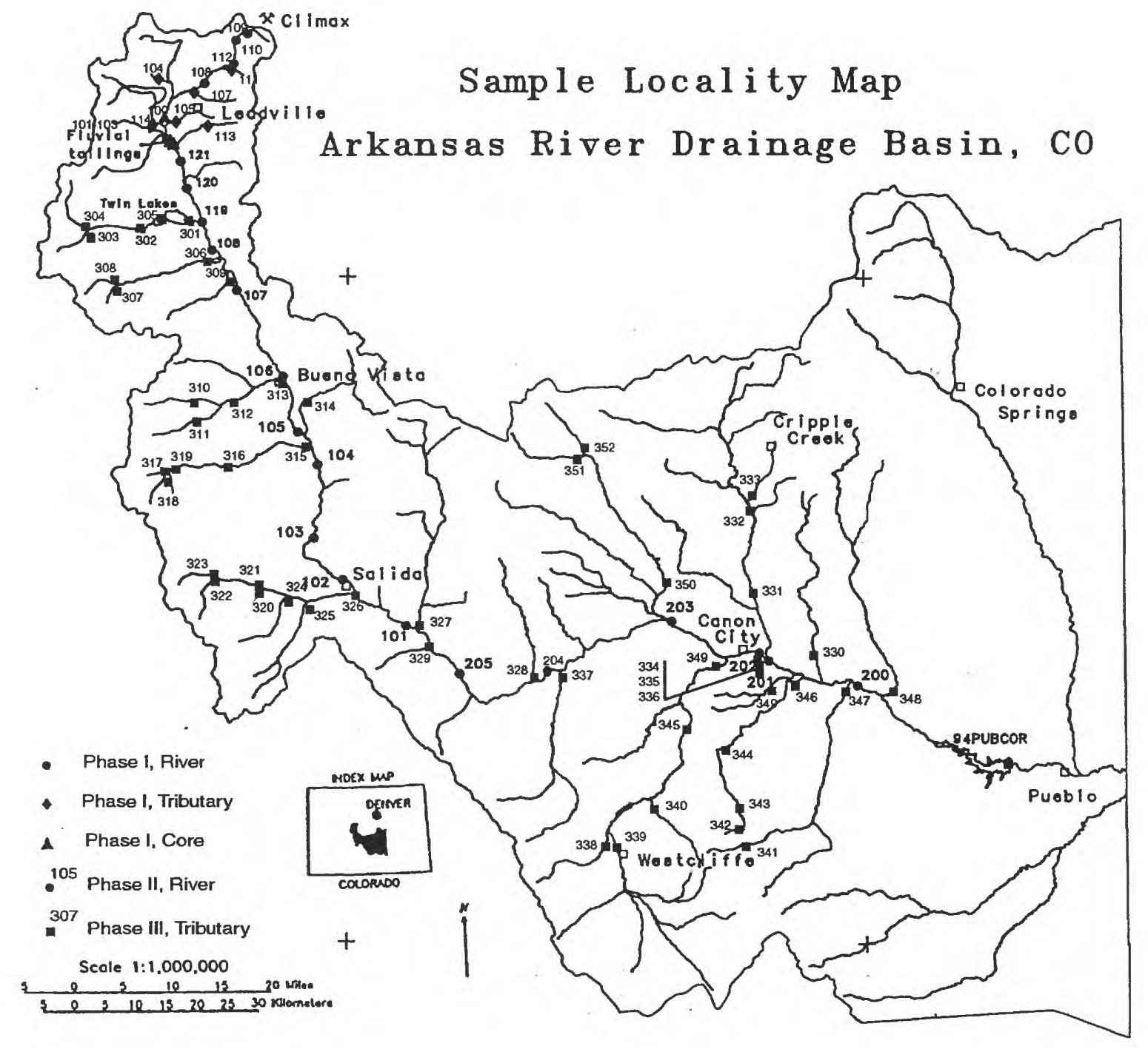

Figure 2. Map of the Arkansas River basin study area showing sample localities for phase I (Church and others, 1993), phase II (Church and others, 1994) and phase III of the study. 
F产

定

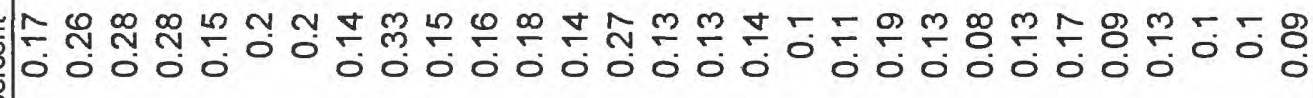

$\xi$

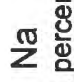

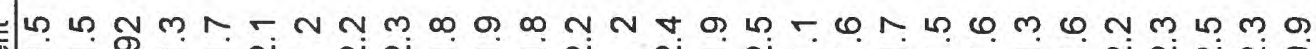

క

잉

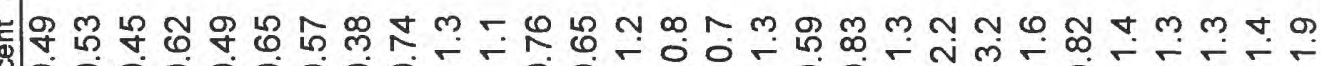

ظे $\checkmark \check{\mathrm{d}}$

Nm- $m$ m m 0 GN

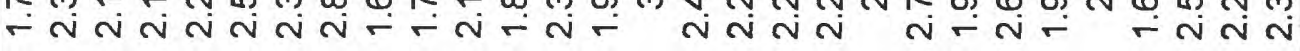

巡

正

A

லั

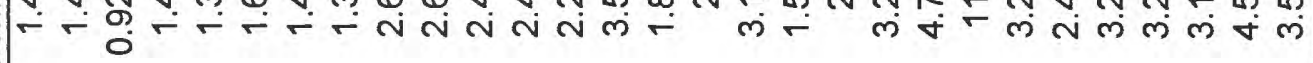

ङ

¿

$\varphi \forall \forall m \infty \forall \infty \sigma \wedge \omega \wedge \omega \varphi \wedge$ m 路

ம்

३

ш

>

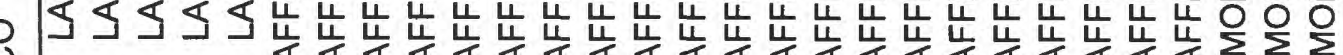

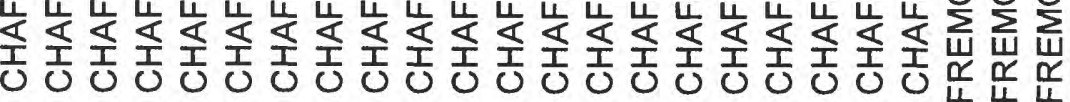

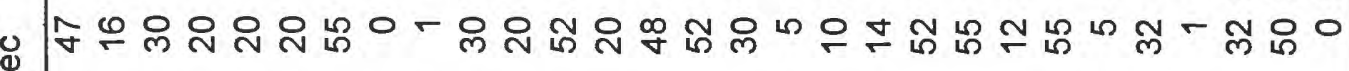
ळ

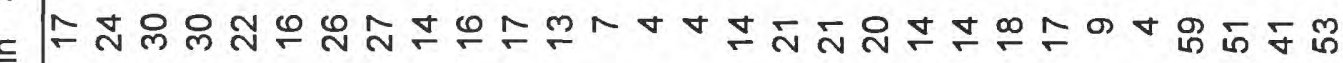
$\sum$

क)

$\emptyset \varphi \emptyset \varphi \varphi \varphi \varphi \varphi \varphi \varphi \varphi \varphi \varphi \varphi \varphi \varphi \varphi \varphi \varphi \varphi \varphi \varphi \varphi \varphi$ น น น

๑ั

४

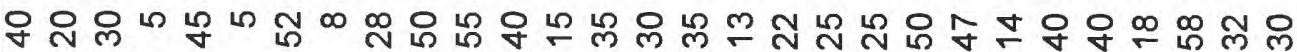

品

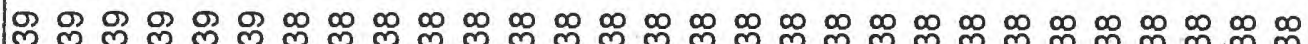

โริ 


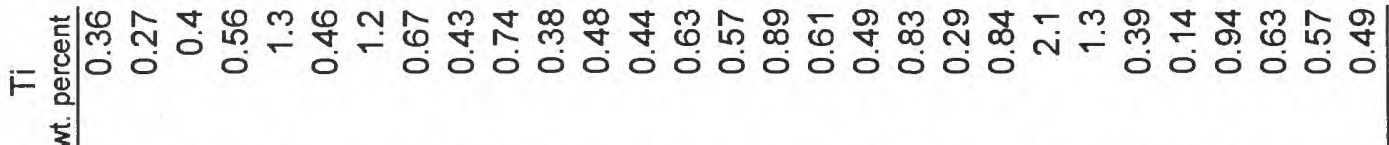

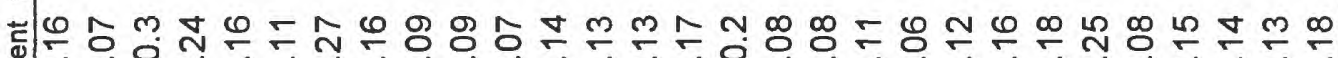
a.

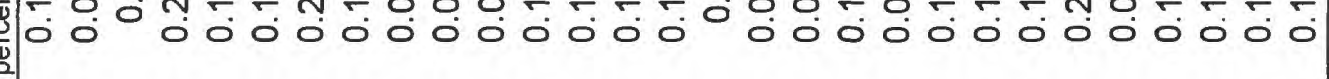
¿

แ

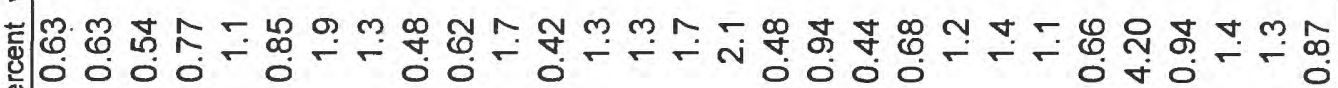
$\sum$ 要

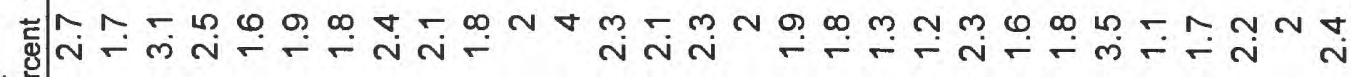
$x \div$

实 눈

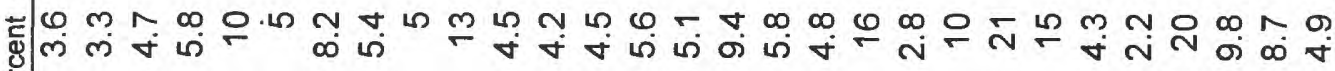
1 잉

ชే

- $N$ แ $\mathcal{0}$

亏 षष्ष

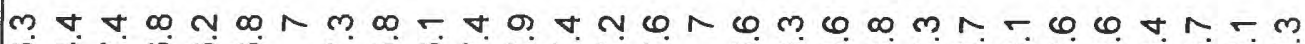
ब

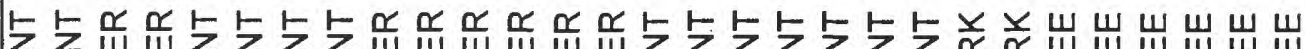
它

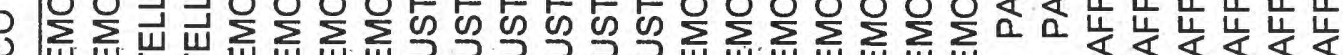
㟧岩

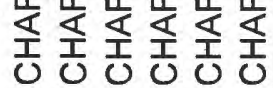
$\infty$ 人 कू

든

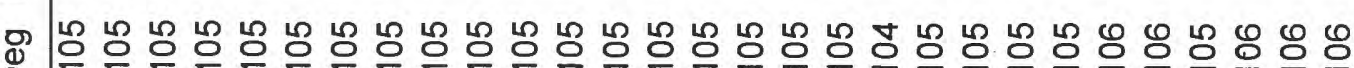

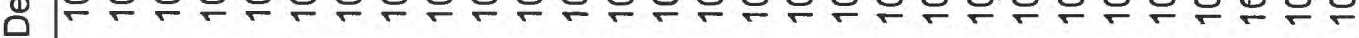
8

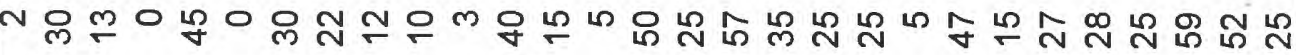

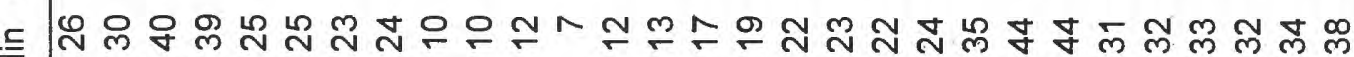

চ ○

-

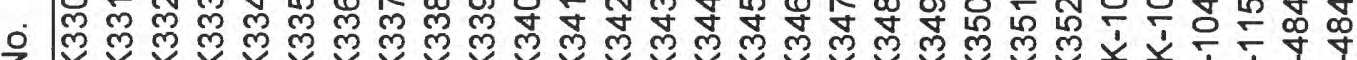

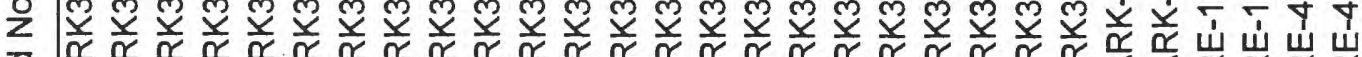

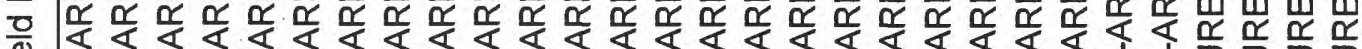

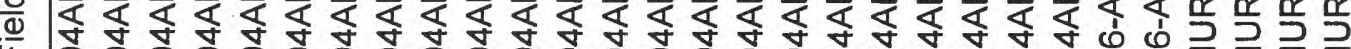




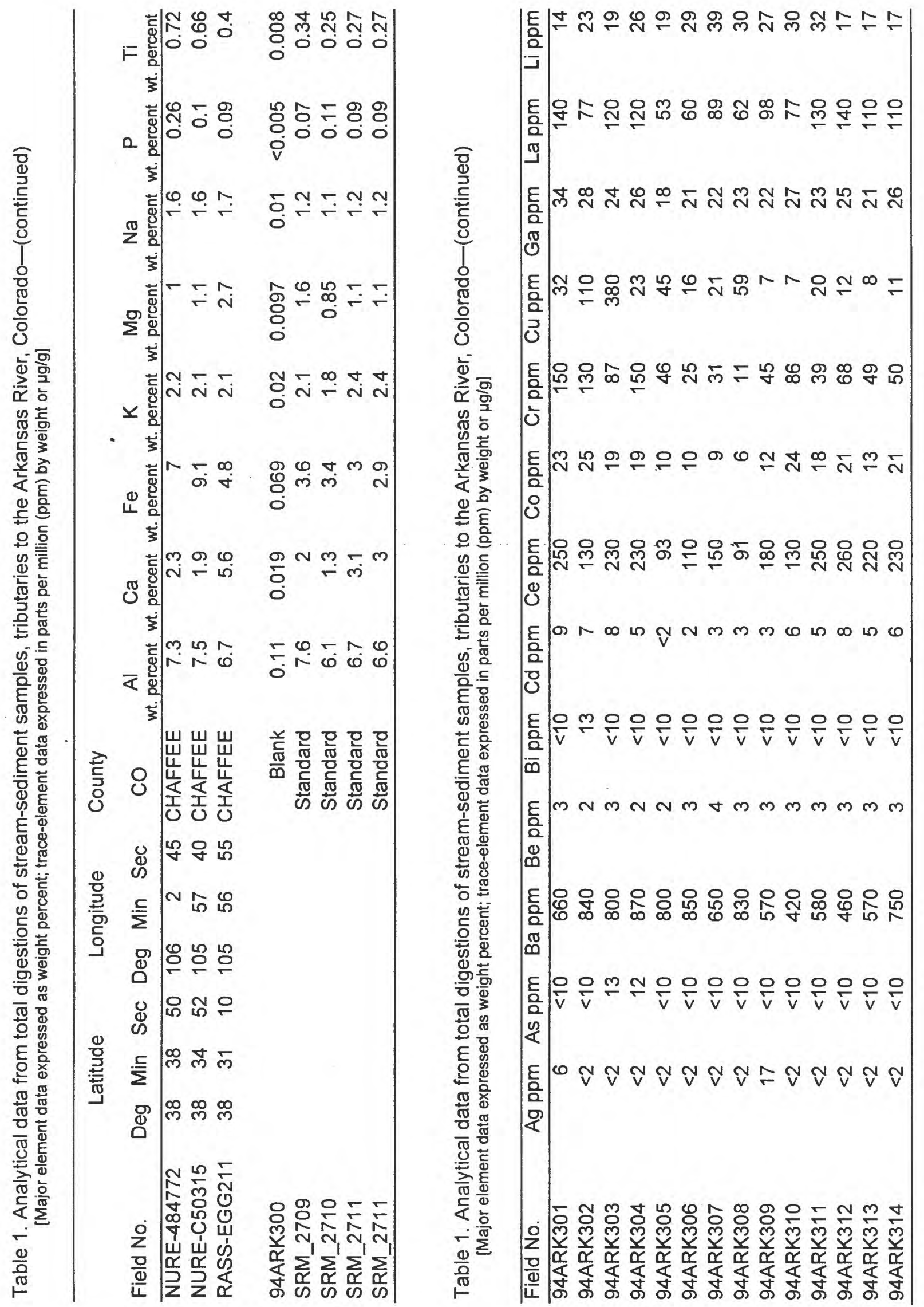




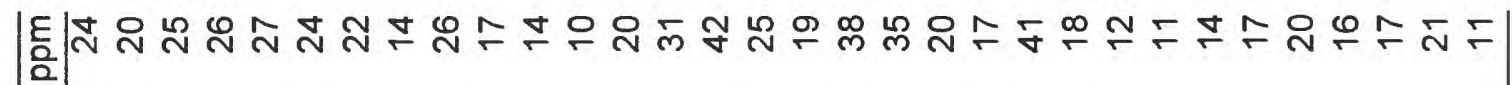

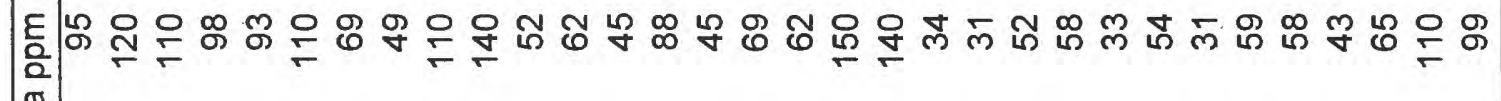
落

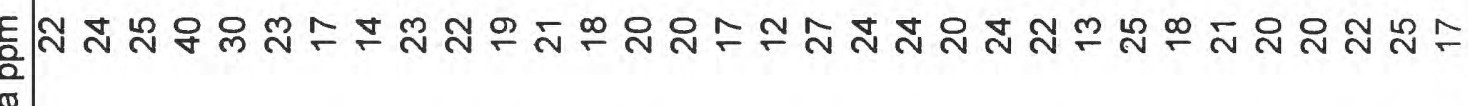
○

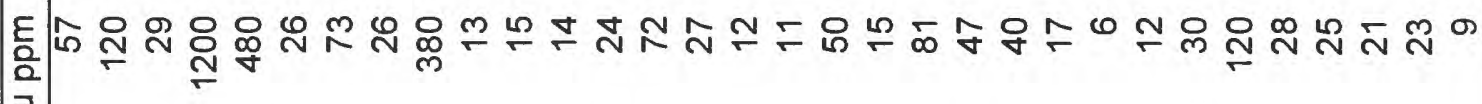
उ.

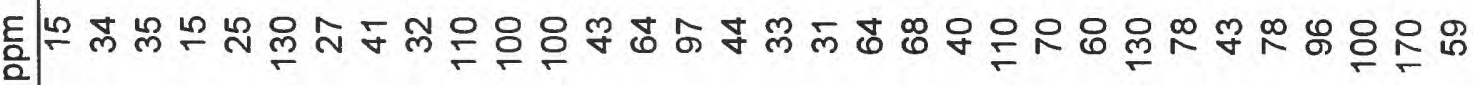

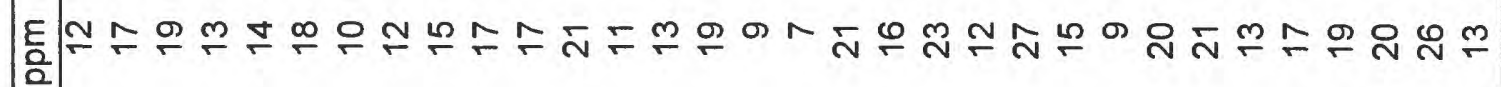
ن

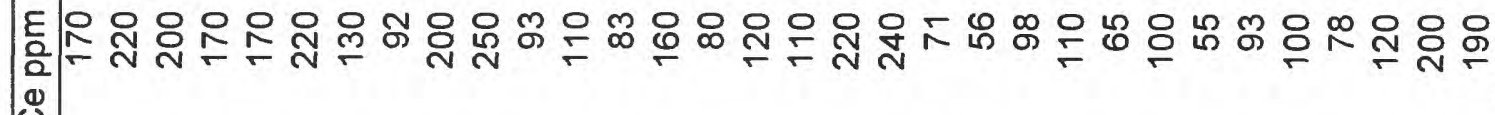

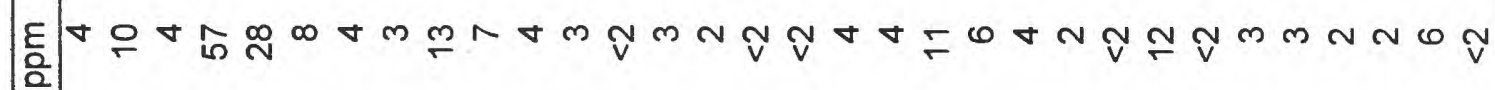
ठ

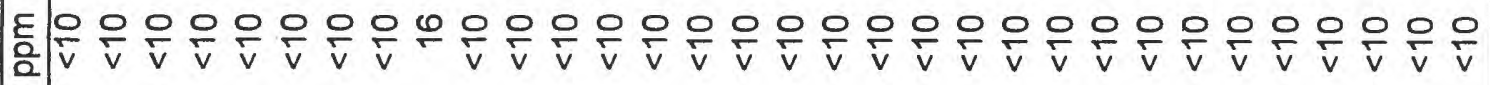
$\bar{\infty}$

Em ఖ

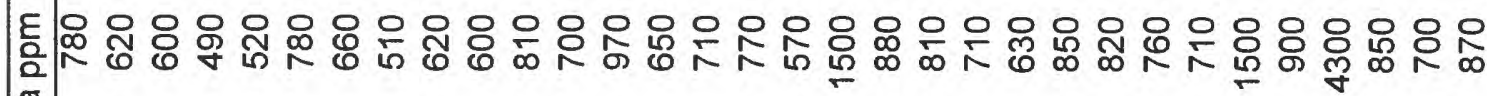
$\stackrel{\pi}{\infty}$

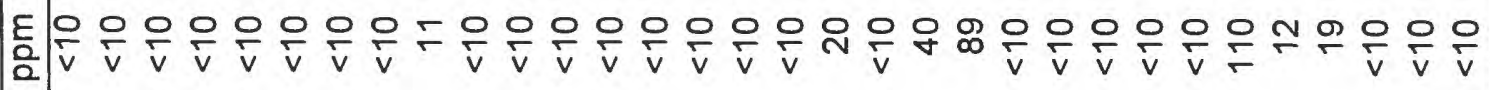
骂

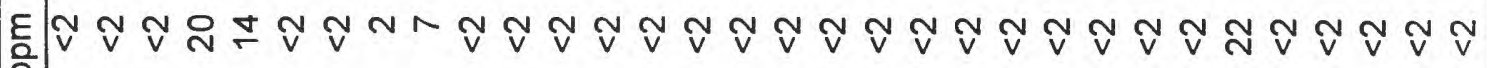
号

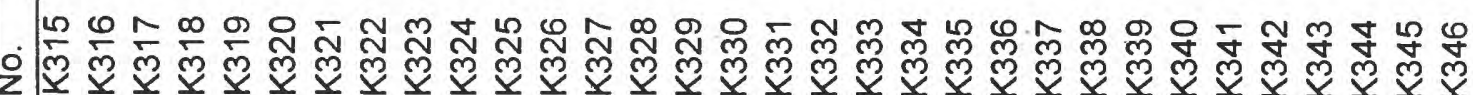

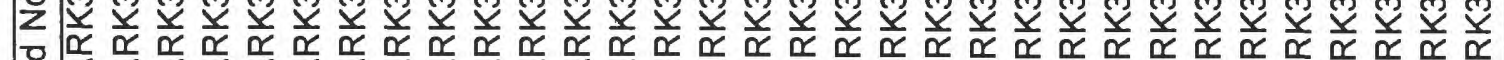

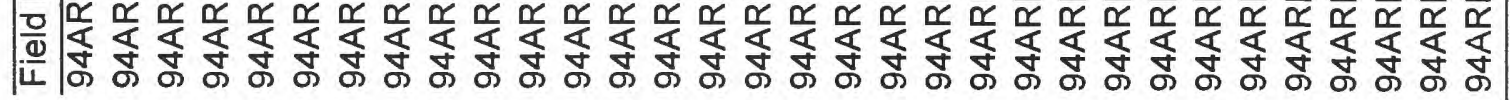




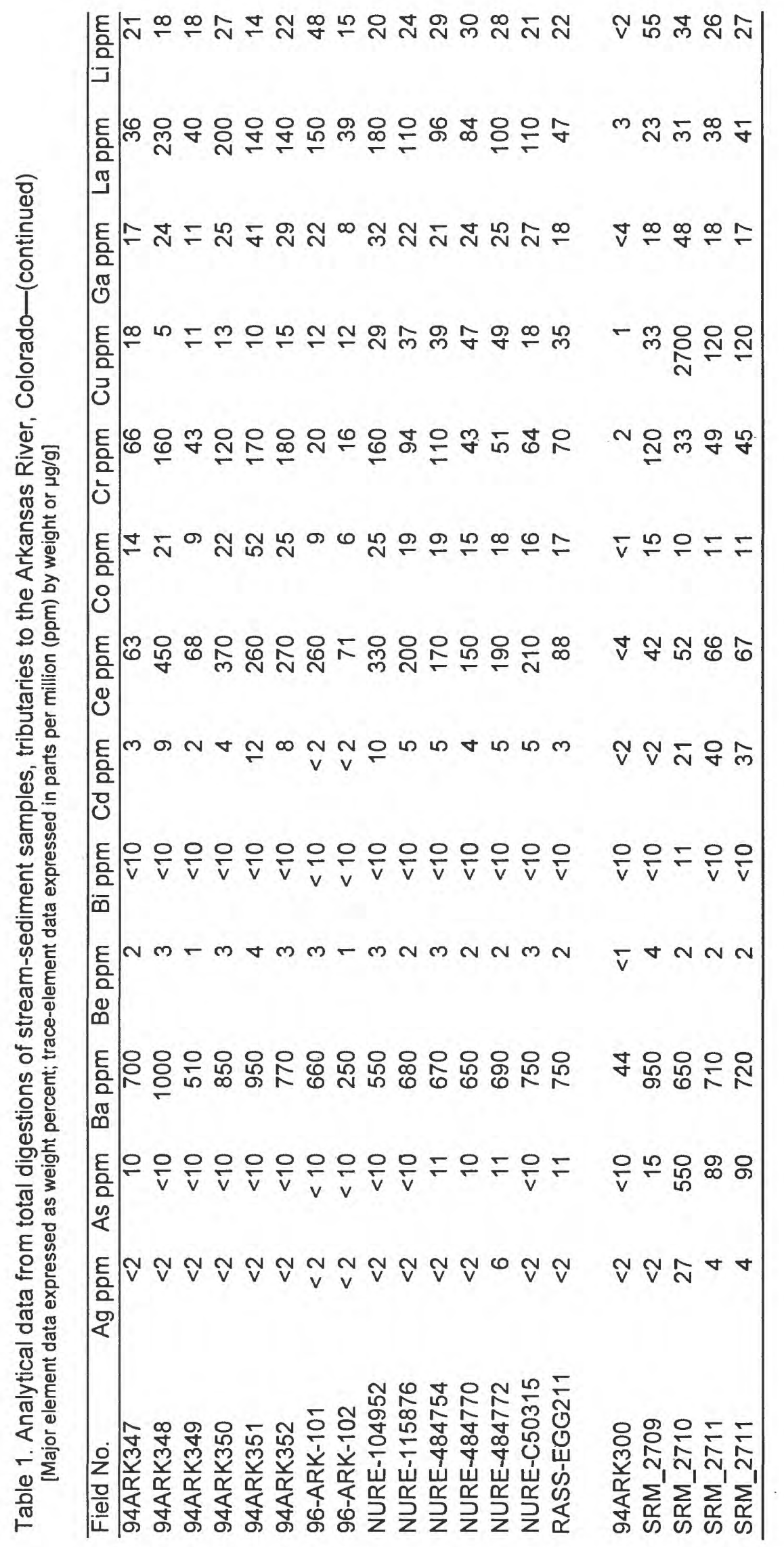




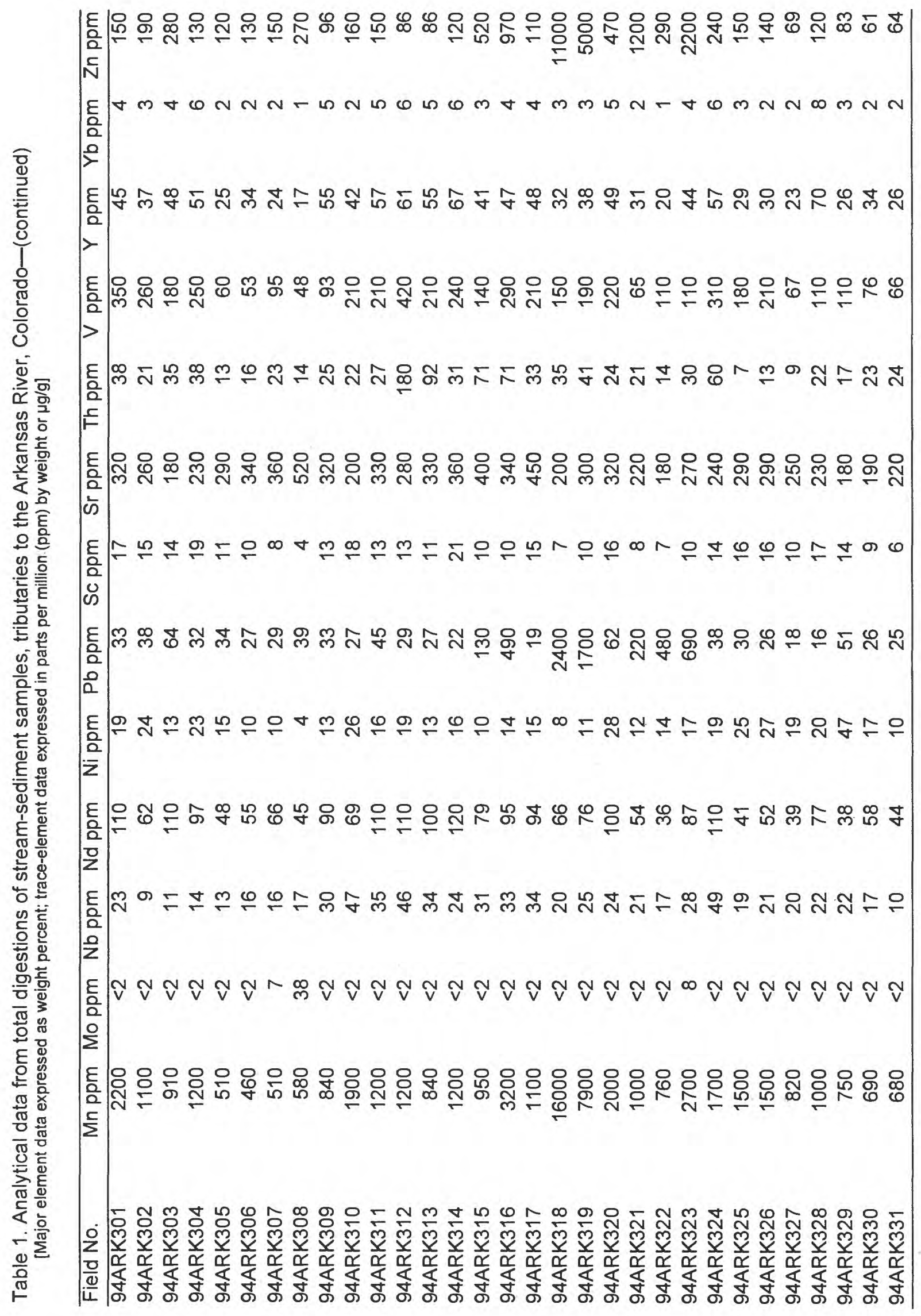




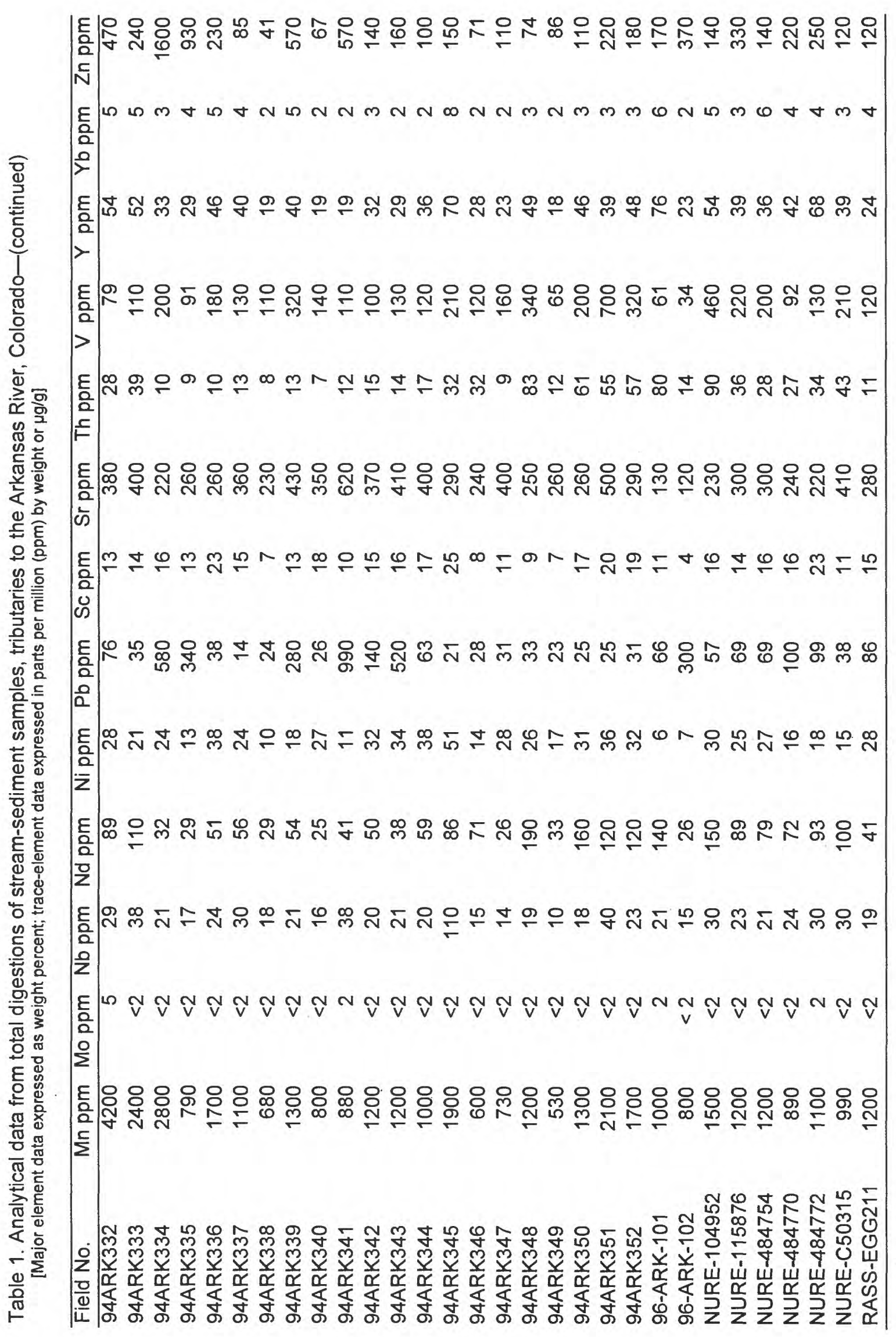




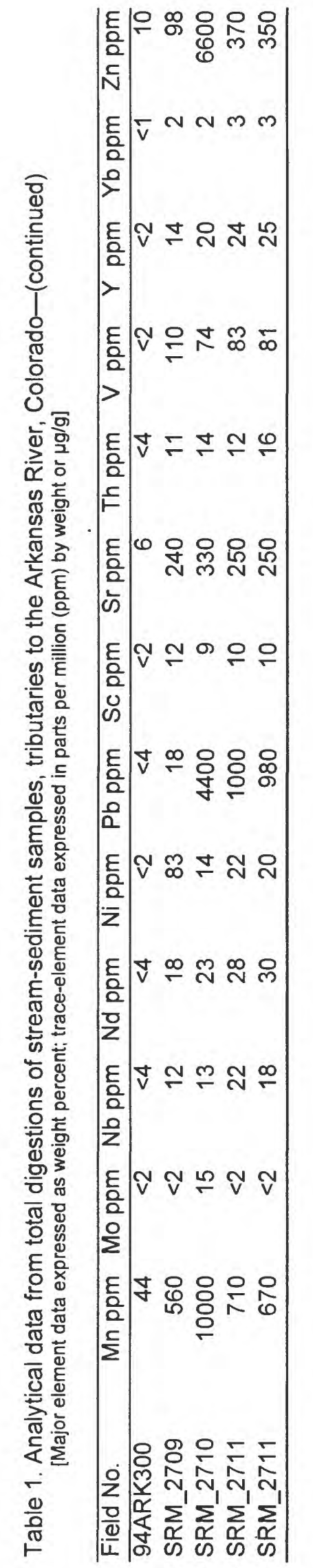

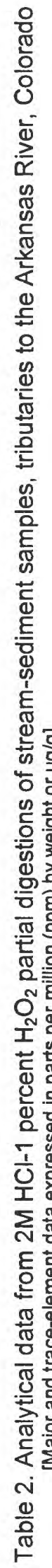

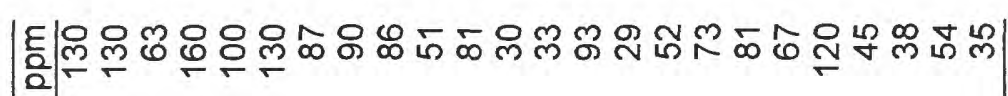
ฌ

ย

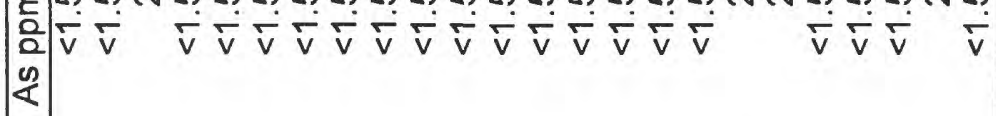

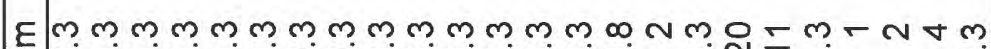
a 운

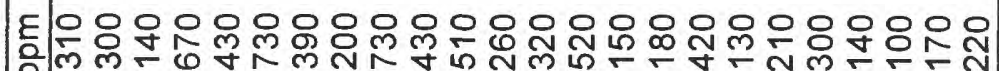
F

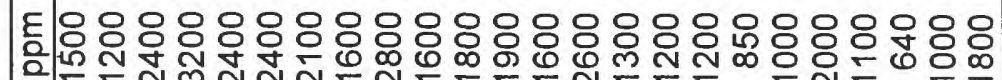
๑

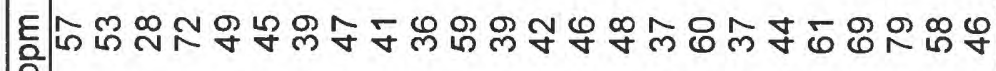
$\stackrel{\pi}{2}$

팅ㅇㅇㅇㅇㅇㅇㅇㅇㅇㅇㅇㅇㅇㅇㅇㅇㅇㅇㅇㅇㅇㅇㅛ

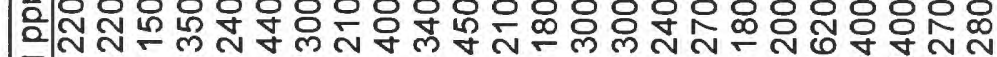
$\sum^{\circ}$

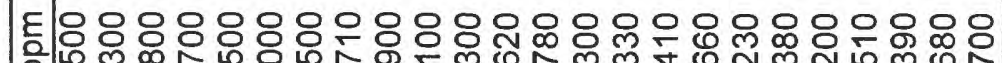

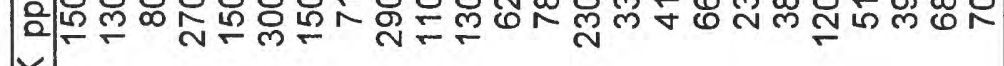

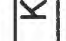

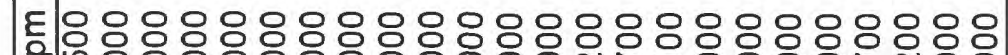
잉

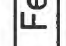

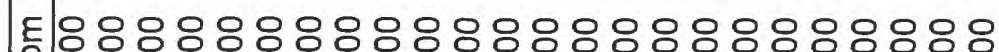

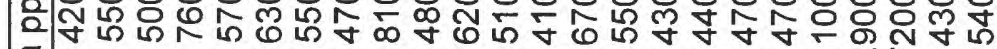
ऽ

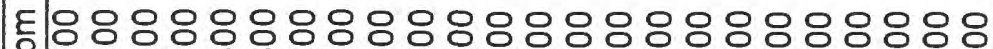

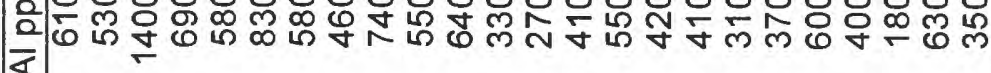
$\bar{\alpha}$

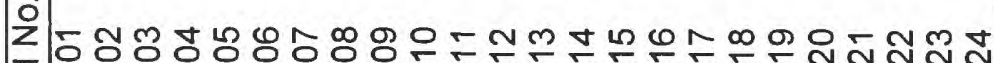


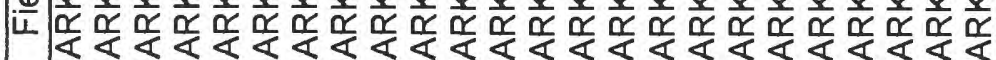

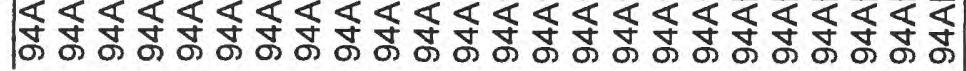




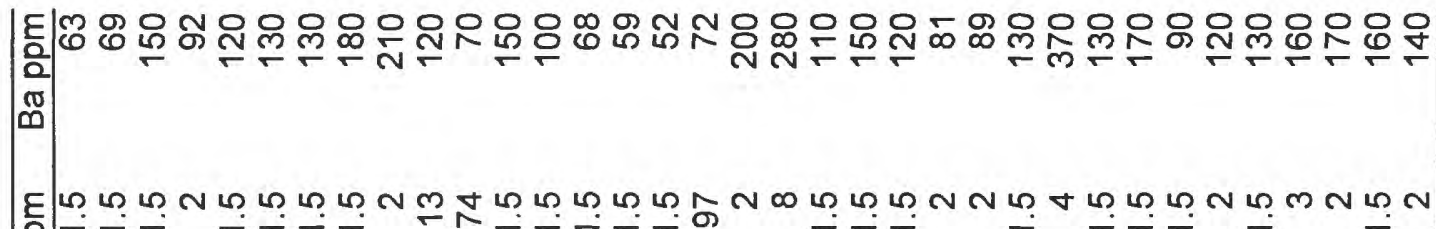

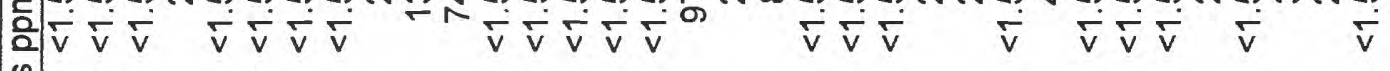
是

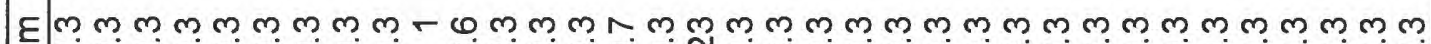
a 安

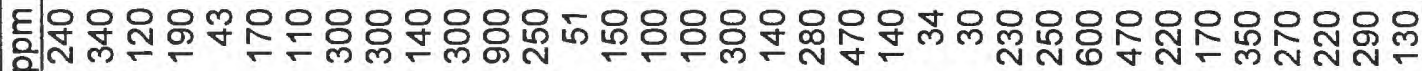
F

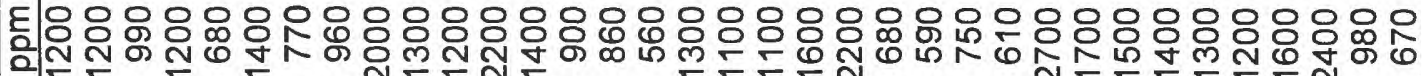
a.

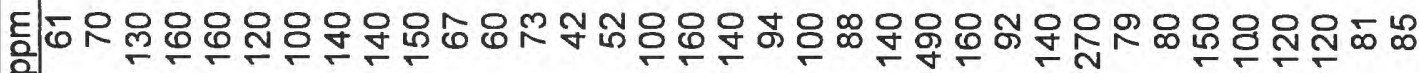
Z

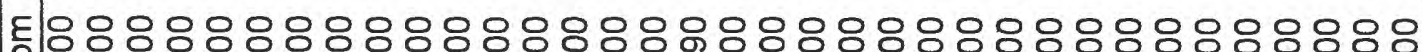

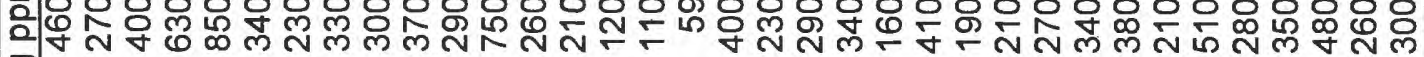
$\stackrel{\text { D) }}{\Sigma}$

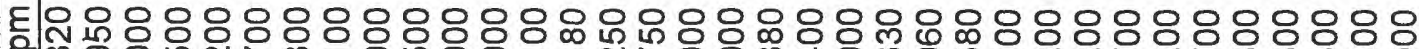
은

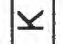

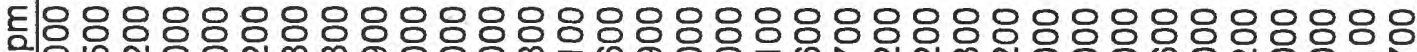

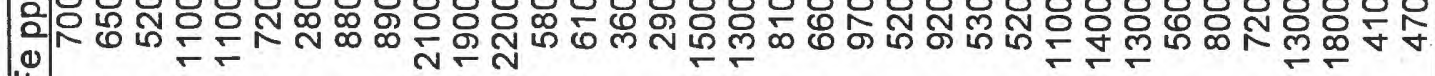
这

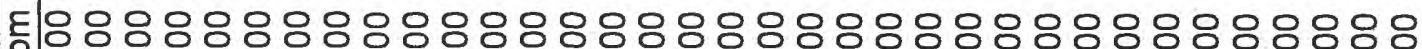

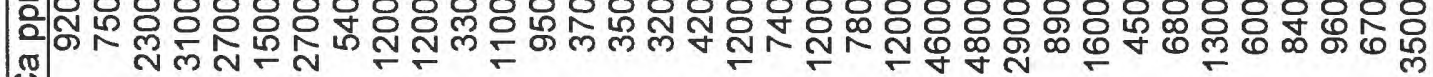
○

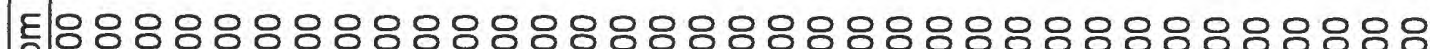

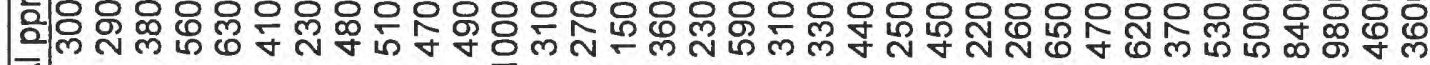
ष

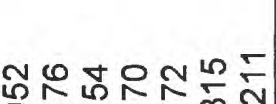

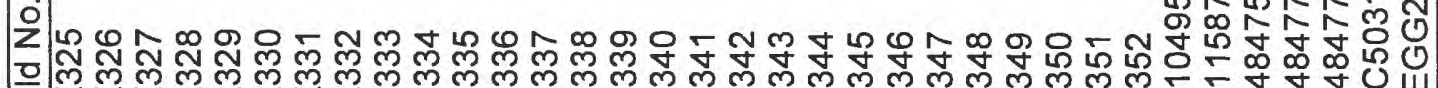
@

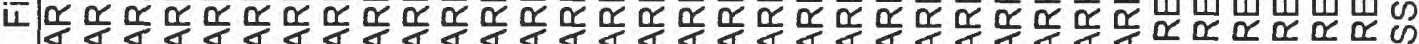

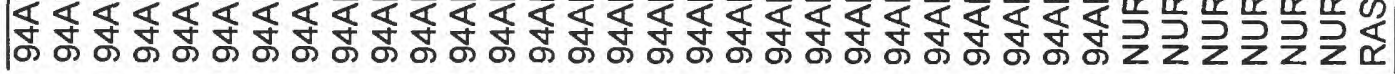



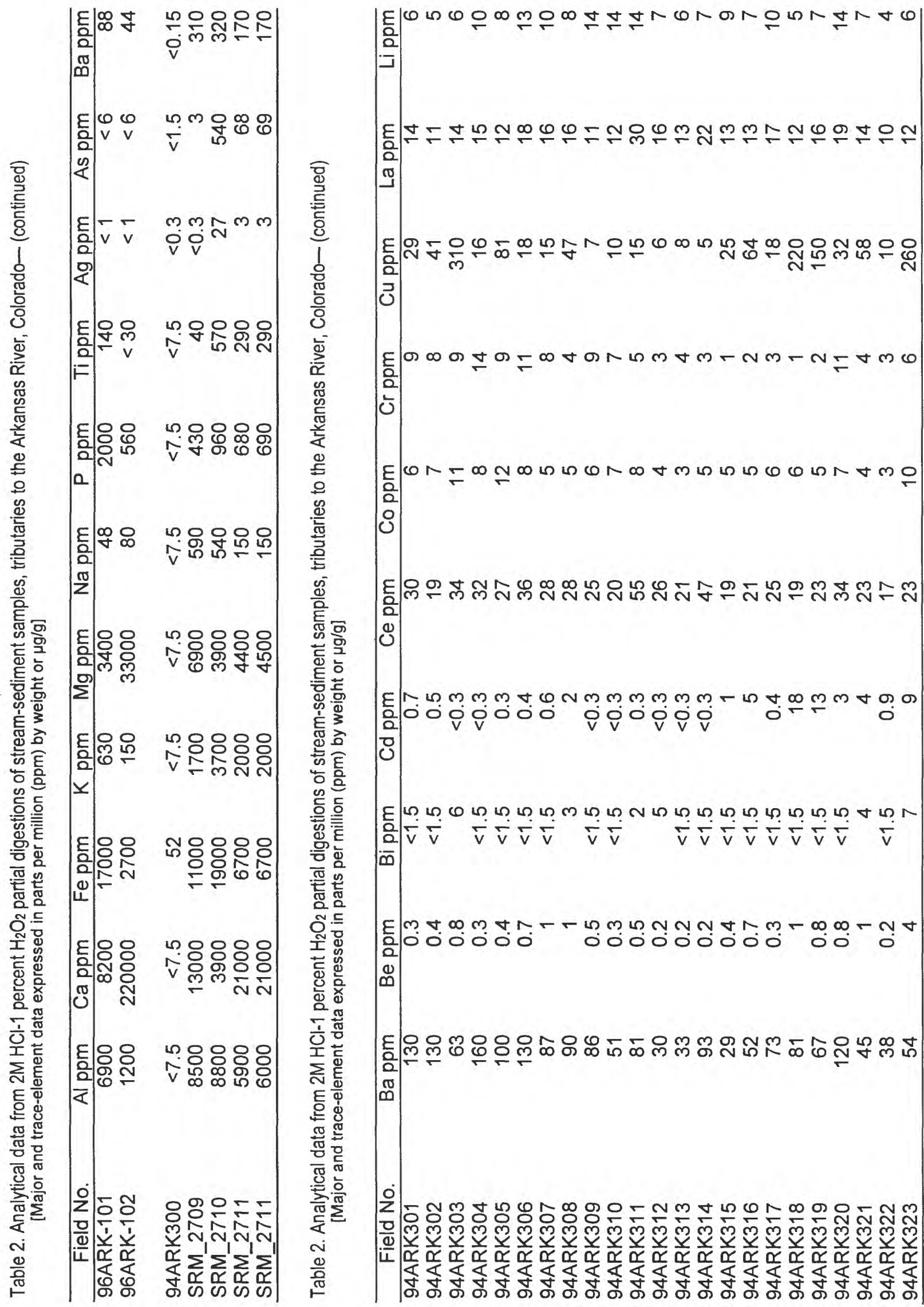


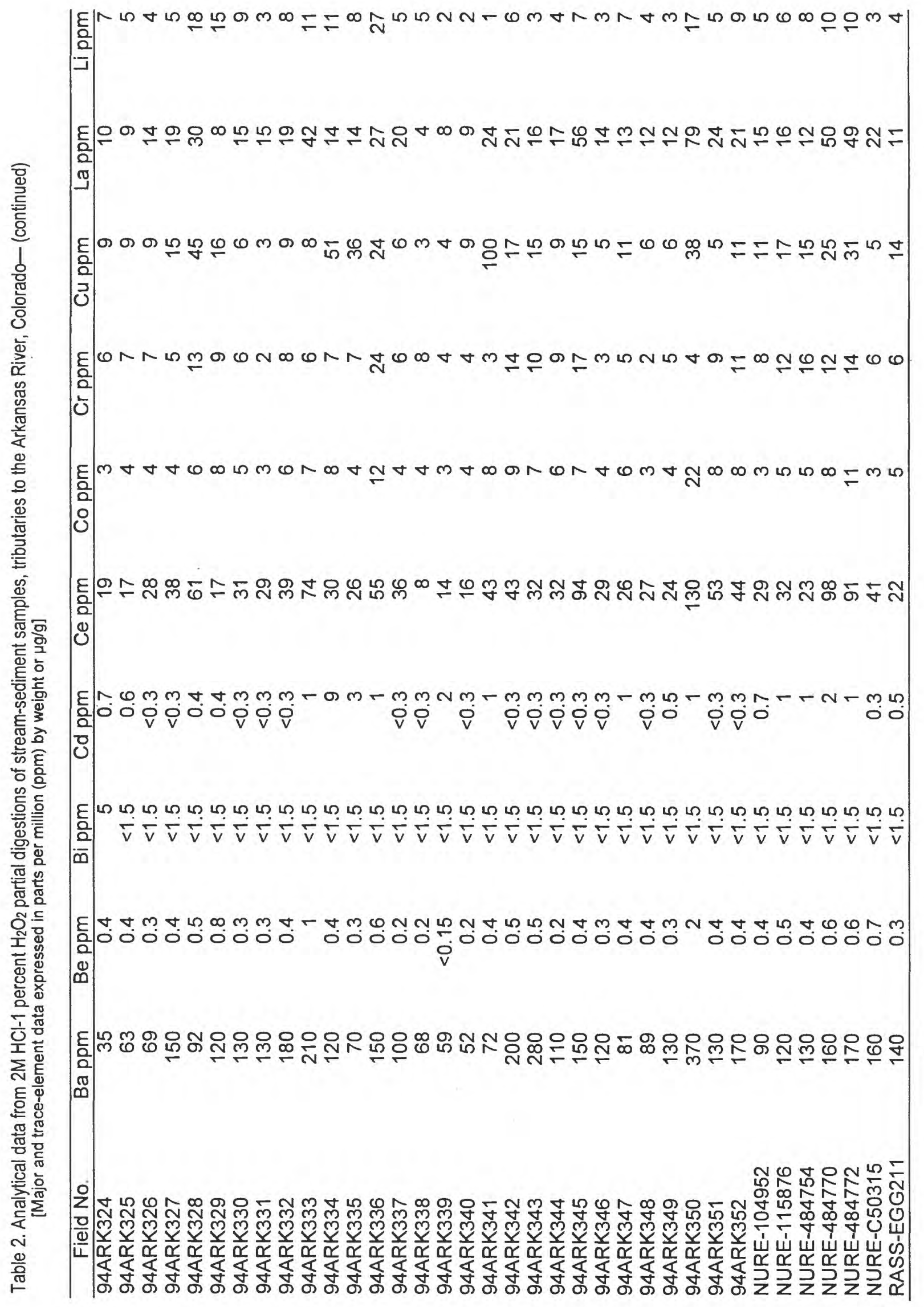




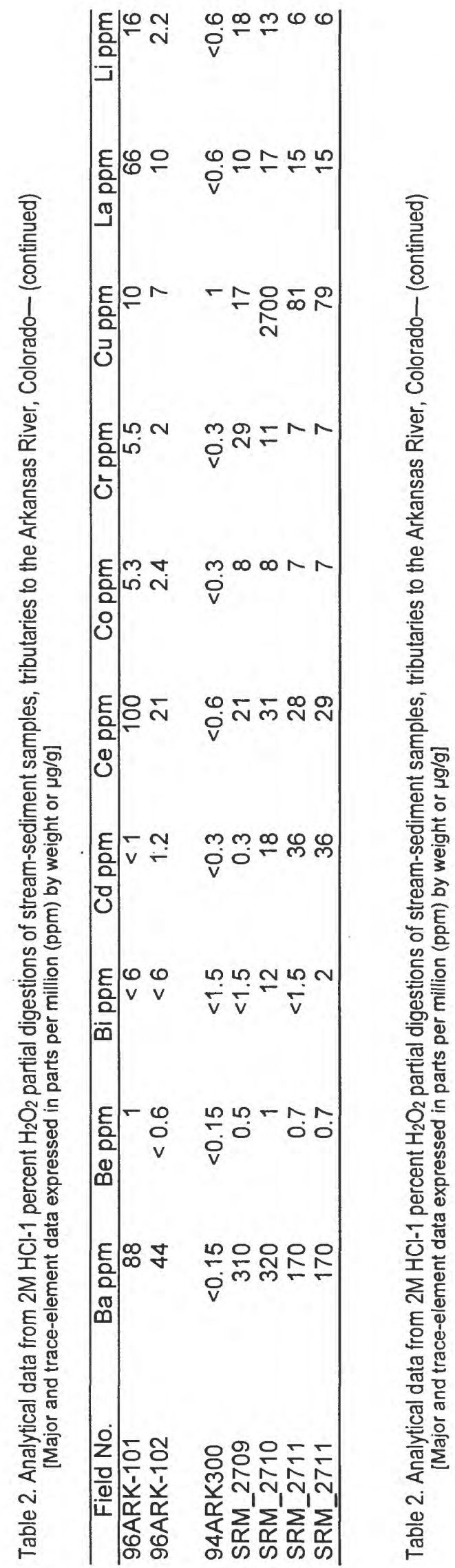

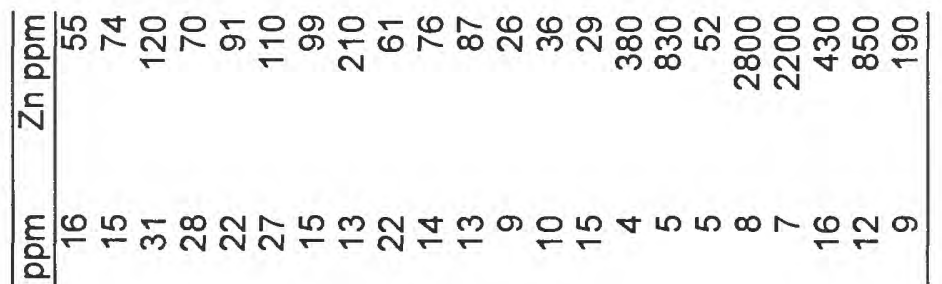

$>$

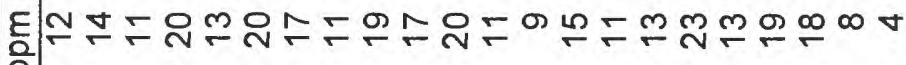

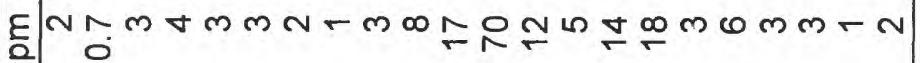

F

要

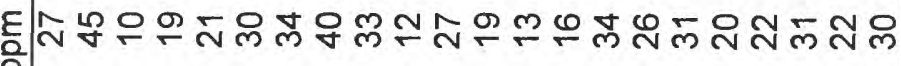

is

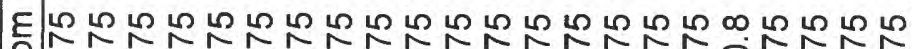

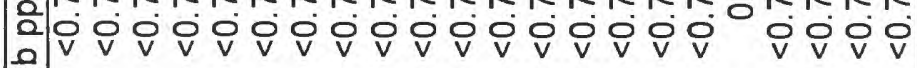

택ำก a

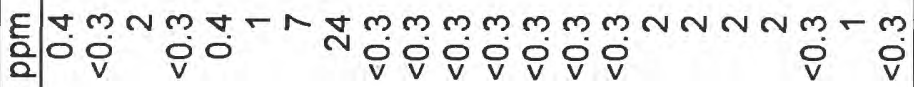

$\stackrel{\circ}{\Sigma}$

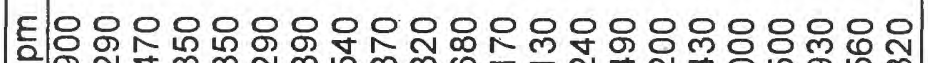

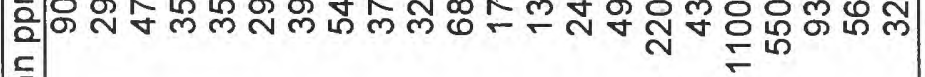

도

인.

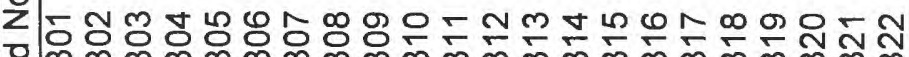

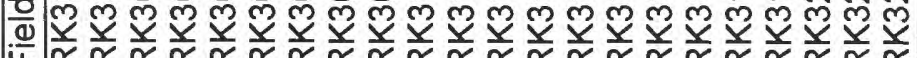

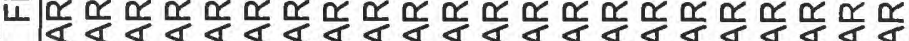

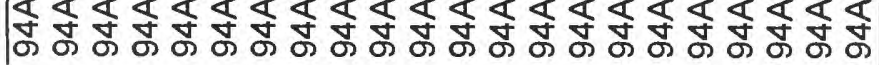




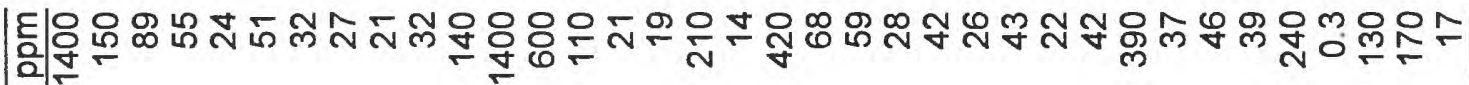
\&

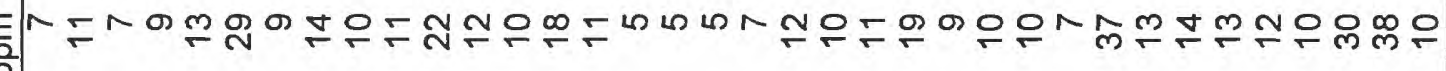

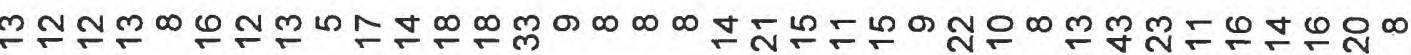

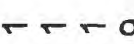

$r+r$

$N m N m N$

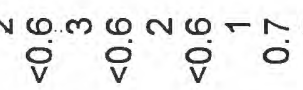

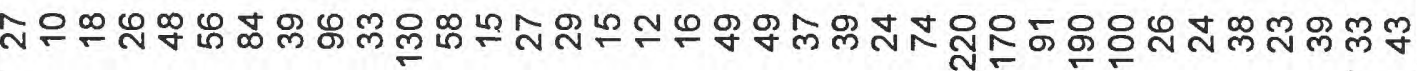

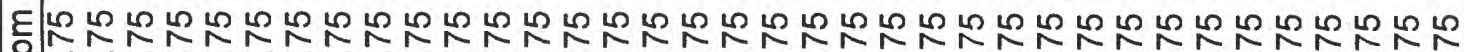
응

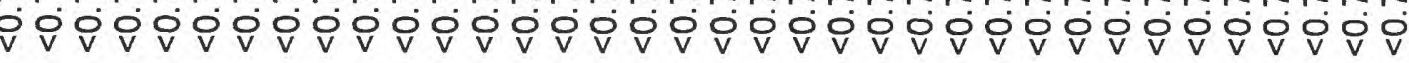

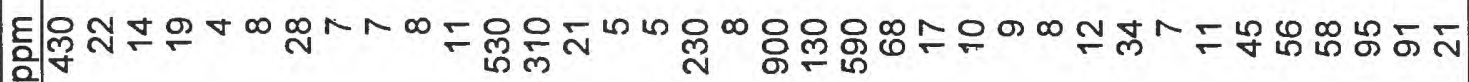
$\frac{0}{0}$

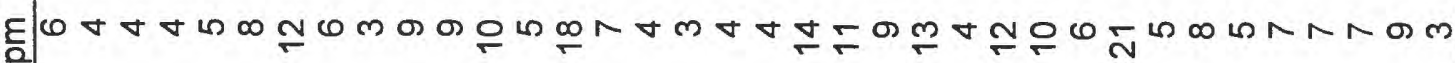

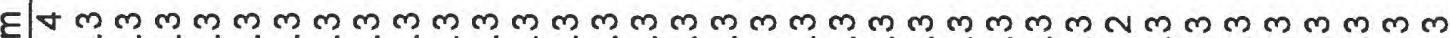
i

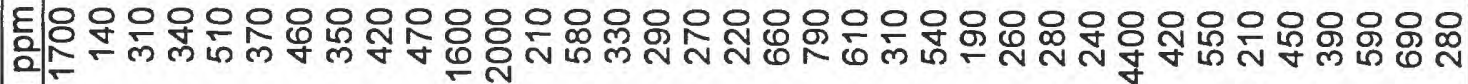




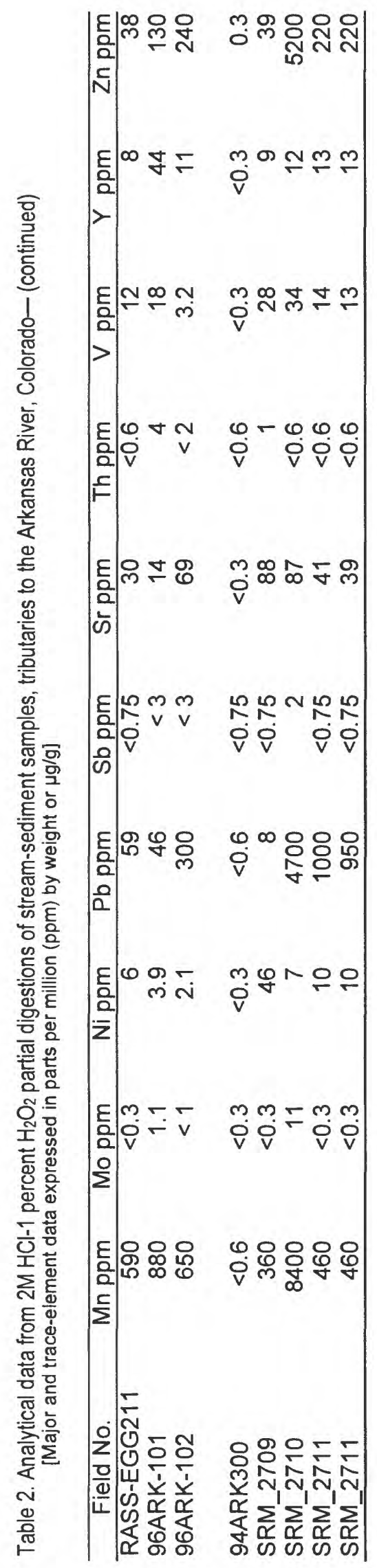


Table 3. Lead isotopic data for selected stream-sediment samples from tributaries, Arkansas River watershed, Colo.

[Pb isotopic data are corrected for mass fractionation of $0.147 \pm 0.03 \%$ (low T) or $0.13 \pm 0.03 \%$ (high T) per mass unit based on analyses of NBS Standard SRM 981 ( 14 analyses of 10 standards)]

\begin{tabular}{|c|c|c|c|c|c|c|c|c|c|c|}
\hline Field No. & $\begin{array}{l}\text { County } \\
\text { Colo. }\end{array}$ & $\frac{206 \mathrm{~Pb}^{\star *}}{204 \mathrm{~Pb}}$ & & $\begin{array}{c}2 \text { sigma } \\
\text { mean }\end{array}$ & $\frac{207 \mathrm{~Pb}}{204 \mathrm{~Pb}}$ & & $\begin{array}{c}2 \text { sigma } \\
\text { mean }\end{array}$ & $\frac{208 \mathrm{~Pb}}{204 \mathrm{~Pb}}$ & & $\begin{array}{c}2 \text { sigma } \\
\text { mean }\end{array}$ \\
\hline 94ARK301 & LAKE & 18.523 & \pm & 0.011 & 15.608 & \pm & 0.014 & 38.520 & \pm & 0.046 \\
\hline 94ARK302 & LAKE & 18.106 & \pm & 0.011 & 15.555 & \pm & 0.014 & 38.255 & \pm & 0.046 \\
\hline 94ARK303 & LAKE & 18.124 & \pm & 0.011 & 15.558 & \pm & 0.014 & 38.287 & \pm & 0.046 \\
\hline 94ARK304 & LAKE & & \pm & & & \pm & & & \pm & \\
\hline 94ARK305 & LAKE & & \pm & & & \pm & & & \pm & \\
\hline 94ARK306 & CHAFFEE & & \pm & & & \pm & & & \pm & \\
\hline 94ARK307 & CHAFFEE & 17.783 & \pm & 0.011 & 15.525 & \pm & 0.014 & 38.207 & \pm & 0.046 \\
\hline 94ARK308 & CHAFFEE & & \pm & & & \pm & & & \pm & \\
\hline 94ARK309 & CHAFFEE & & \pm & & & \pm & & & \pm & \\
\hline 94ARK310 & CHAFFEE & & \pm & & & \pm & & & \pm & \\
\hline 94ARK311 & CHAFFEE & 17.985 & \pm & 0.011 & 15.536 & \pm & 0.014 & 38.211 & \pm & 0.046 \\
\hline 94ARK312 & CHAFFEE & & \pm & & & \pm & & & \pm & \\
\hline 94ARK313 & CHAFFEE & & \pm & & & \pm & & & \pm & \\
\hline 94ARK314 & CHAFFEE & 19.579 & \pm & 0.012 & 15.698 & \pm & 0.014 & 40.048 & \pm & 0.048 \\
\hline 94ARK315 & CHAFFEE & 17.820 & \pm & 0.011 & 15.521 & \pm & 0.014 & 38.100 & \pm & 0.046 \\
\hline 94ARK316 & CHAFFEE & 17.816 & \pm & 0.011 & 15.522 & \pm & 0.014 & 38.098 & \pm & 0.046 \\
\hline 94ARK316D & CHAFFEE & 17.811 & \pm & 0.011 & 15.516 & \pm & 0.014 & 38.077 & \pm & 0.046 \\
\hline 94ARK317 & CHAFFEE & 18.020 & \pm & 0.011 & 15.552 & \pm & 0.014 & 38.280 & \pm & 0.046 \\
\hline 94ARK318 & CHAFFEE & 17.806 & \pm & 0.011 & 15.519 & \pm & 0.014 & 38.082 & \pm & 0.046 \\
\hline 94ARK319 & CHAFFEE & 17.808 & \pm & 0.011 & 15.520 & \pm & 0.014 & 38.088 & \pm & 0.046 \\
\hline 94ARK320 & CHAFFEE & 19.280 & \pm & 0.012 & 15.648 & \pm & 0.014 & 38.584 & \pm & 0.047 \\
\hline 94ARK321 & CHAFFEE & 18.725 & \pm & 0.011 & 15.606 & \pm & 0.014 & 38.473 & \pm & 0.046 \\
\hline 94ARK322 & CHAFFEE & 19.516 & \pm & 0.012 & 15.682 & \pm & 0.014 & 38.950 & \pm & 0.047 \\
\hline 94ARK323 & CHAFFEE & 17.938 & \pm & 0.011 & 15.517 & \pm & 0.014 & 37.872 & \pm & 0.046 \\
\hline 94ARK323D & CHAFFEE & 17.953 & \pm & 0.011 & 15.535 & \pm & 0.014 & 37.931 & \pm & 0.046 \\
\hline 94ARK324 & CHAFFEE & 18.950 & \pm & 0.012 & 15.618 & \pm & 0.014 & 38.496 & \pm & 0.046 \\
\hline 94ARK325 & CHAFFEE & 19.081 & \pm & 0.012 & 15.649 & \pm & 0.014 & 38.426 & \pm & 0.046 \\
\hline 94ARK326 & CHAFFEE & & \pm & & & \pm & & & \pm & \\
\hline 94ARK327 & FREMONT & 24.028 & \pm & 0.015 & 16.091 & \pm & 0.015 & 40.612 & \pm & 0.049 \\
\hline 94ARK328 & FREMONT & 23.759 & \pm & 0.014 & 16.074 & \pm & 0.015 & 40.530 & \pm & 0.049 \\
\hline 94ARK329 & FREMONT & 19.312 & \pm & 0.012 & 15.683 & \pm & 0.014 & 38.467 & \pm & 0.046 \\
\hline 94ARK330 & FREMONT & & \pm & & & \pm & & & \pm & \\
\hline 94ARK331 & FREMONT & & \pm & & & \pm & & & \pm & \\
\hline 94ARK332 & TELLER & 19.589 & \pm & 0.012 & 15.708 & \pm & 0.014 & 39.244 & \pm & 0.047 \\
\hline 94ARK333 & TELLER & 19.621 & \pm & 0.012 & 15.692 & \pm & 0.014 & 39.367 & \pm & 0.047 \\
\hline 94ARK334 & FREMONT & 17.877 & \pm & 0.011 & 15.539 & \pm & 0.014 & 38.424 & \pm & 0.047 \\
\hline 94ARK335 & FREMONT & 17.941 & \pm & 0.011 & 15.544 & \pm & 0.014 & 38.445 & \pm & 0.046 \\
\hline 94ARK336 & FREMONT & 19.380 & \pm & 0.012 & 15.679 & \pm & 0.014 & 38.592 & \pm & 0.046 \\
\hline 94ARK337 & FREMONT & & \pm & & & \pm & & & \pm & \\
\hline 94ARK338 & CUSTER & 19.350 & \pm & 0.013 & 15.652 & \pm & 0.015 & 38.163 & \pm & 0.047 \\
\hline 94ARK339 & CUSTER & 17.447 & \pm & 0.011 & 15.468 & \pm & 0.014 & 37.070 & \pm & 0.045 \\
\hline 94ARK340 & CUSTER & & \pm & & & \pm & & & \pm & \\
\hline
\end{tabular}


Table 3. Lead isotopic data for selected stream-sediment samples from tributaries, Arkansas River watershed, Colo.-(continued) $[\mathrm{Pb}$ isotopic data are corrected for mass fractionation of $0.147 \pm 0.03 \%$ (low $\mathrm{T}$ ) or $0.13 \pm 0.03 \%$ (high $\mathrm{T}$ ) per mass unit based on analyses of NBS Standard SRM 981 ( 14 analyses of 10 standards)]

\begin{tabular}{|c|c|c|c|c|c|c|c|c|c|c|}
\hline Field No. & $\begin{array}{l}\text { County } \\
\text { Colo. }\end{array}$ & $\frac{206 \mathrm{~Pb}^{\star *}}{204 \mathrm{~Pb}}$ & & $\begin{array}{c}2 \text { sigma } \\
\text { mean }\end{array}$ & $\frac{207 \mathrm{~Pb}}{204 \mathrm{~Pb}}$ & & $\begin{array}{c}2 \text { sigma } \\
\text { mean }\end{array}$ & $\frac{208 \mathrm{~Pb}}{204 \mathrm{~Pb}}$ & & $\begin{array}{c}2 \text { sigma } \\
\text { mean }\end{array}$ \\
\hline 94ARK341 & CUSTER & 17.594 & \pm & 0.013 & 15.476 & \pm & 0.015 & 37.432 & \pm & 0.047 \\
\hline 94ARK342 & CUSTER & 17.140 & \pm & 0.011 & 15.457 & \pm & 0.014 & 38.052 & \pm & 0.046 \\
\hline 94ARK343 & CUSTER & 17.055 & \pm & 0.010 & 15.445 & \pm & 0.014 & 37.983 & \pm & 0.046 \\
\hline 94ARK344 & FREMONT & 17.190 & \pm & 0.010 & 15.465 & \pm & 0.014 & 38.087 & \pm & 0.046 \\
\hline 94ARK345 & FREMONT & 18.280 & \pm & 0.011 & 15.548 & \pm & 0.014 & 38.719 & \pm & 0.047 \\
\hline 94ARK346 & FREMONT & & \pm & & & \pm & & & \pm & \\
\hline 94ARK347 & FREMONT & 19.365 & \pm & 0.012 & 15.681 & \pm & 0.014 & 39.178 & \pm & 0.047 \\
\hline 94ARK348 & FREMONT & & \pm & & & \pm & & & \pm & \\
\hline 94ARK349 & FREMONT & & \pm & & & \pm & & & \pm & \\
\hline 94ARK350 & FREMONT & 19.574 & \pm & 0.012 & 15.688 & \pm & 0.014 & 39.205 & \pm & 0.047 \\
\hline 94ARK351 & PARK & 19.844 & \pm & 0.012 & 15.722 & \pm & 0.014 & 38.998 & \pm & 0.047 \\
\hline 94ARK352 & PARK & 18.948 & \pm & 0.012 & 15.635 & \pm & 0.015 & 38.709 & \pm & 0.047 \\
\hline 96ARK101 & LAKE & 20.880 & \pm & 0.013 & 15.808 & \pm & 0.014 & 39.860 & \pm & 0.048 \\
\hline 96ARK102 & LAKE & 19.856 & \pm & 0.012 & 15.880 & \pm & 0.014 & 39.276 & \pm & 0.047 \\
\hline ARK TY-S1 & LAKE & 18.049 & \pm & 0.011 & 15.556 & \pm & 0.014 & 38.148 & \pm & 0.046 \\
\hline ARK TY-S13 & LAKE & 17.965 & \pm & 0.011 & 15.540 & \pm & 0.014 & 38.093 & \pm & 0.046 \\
\hline JAY718 & & 20.414 & \pm & 0.012 & 15.796 & \pm & 0.014 & 39.454 & \pm & 0.047 \\
\hline JAY735 & & 40.654 & \pm & 0.026 & 17.596 & \pm & 0.016 & 44.394 & \pm & 0.054 \\
\hline JAY751 & & 30.207 & \pm & 0.019 & 16.674 & \pm & 0.015 & 41.800 & \pm & 0.050 \\
\hline $93 L V-110$ & LAKE & 18.985 & \pm & 0.012 & 15.639 & \pm & 0.014 & 39.036 & \pm & 0.047 \\
\hline 93LV-112 & LAKE & 18.598 & \pm & 0.011 & 15.615 & \pm & 0.014 & 38.778 & \pm & 0.047 \\
\hline NURE-104952 & CHAFFEE & 17.843 & \pm & 0.011 & 15.527 & \pm & 0.014 & 37.706 & \pm & 0.045 \\
\hline NURE-115876 & CHAFFEE & 18.080 & \pm & 0.011 & 15.549 & \pm & 0.014 & 38.094 & \pm & 0.046 \\
\hline NURE-484754 & CHAFFEE & 17.801 & \pm & 0.011 & 15.524 & \pm & 0.014 & 37.584 & \pm & 0.045 \\
\hline NURE-484770 & CHAFFEE & 18.272 & \pm & 0.011 & 15.568 & \pm & 0.014 & 38.152 & \pm & 0.046 \\
\hline NURE-484772 & CHAFFEE & 18.340 & \pm & 0.011 & 15.574 & \pm & 0.014 & 38.263 & \pm & 0.046 \\
\hline NURE-C50315 & CHAFFEE & 17.965 & \pm & 0.011 & 15.539 & \pm & 0.014 & 37.880 & \pm & 0.046 \\
\hline RASS-EGG211 & CHAFFEE & 18.115 & \pm & 0.011 & 15.546 & \pm & 0.014 & 37.851 & \pm & 0.046 \\
\hline SRM_2709 & Standard & 19.082 & \pm & 0.012 & 15.665 & \pm & 0.014 & 38.931 & \pm & 0.047 \\
\hline SRM_2710 & Standard & 17.831 & \pm & 0.011 & 15.547 & \pm & 0.014 & 38.171 & \pm & 0.046 \\
\hline SRM_2711 & Standard & 17.124 & \pm & 0.011 & 15.454 & \pm & 0.014 & 37.016 & \pm & 0.045 \\
\hline SRM_2711 & Standard & 17.095 & \pm & 0.011 & 15.429 & \pm & 0.014 & 36.922 & \pm & 0.045 \\
\hline
\end{tabular}




\section{References Cited}

Church, S.E., Holmes, C.W., Briggs, P.H., Vaughn, R.B., Cathcart, James, and Marot, Margaret, 1993, Geochemical and lead-isotope data from stream and lake sediments, and cores from the upper Arkansas River drainage: Effects of mining at Leadville, Colorado on heavy-metal concentrations in the Arkansas River: U.S. Geological Survey Open-File Report 93-534, 61 p.

Church, S.E., Wilson, S.A., Vaughn, R.B., and Fey, D.L., 1994, Geochemical and lead isotopic studies of river and lake sediments, upper Arkansas River basin, Twin Lakes to Pueblo, Colorado: U.S. Geological Survey Open-File Report 94-412, 44 p.

Fortescue, J.A.C., 1992, Landscape geochemistry: retrospect and prospect--1990: Applied Geochemistry, v. 7, p. $1-54$.

Smith, S.M., 1994, Geochemical maps of copper, lead, and zinc, upper Arkansas River drainage basin, Colorado: U.S. Geological Survey Open-File Report 94-408, 15 p.

Smith, S.M., 2000, National Geochemical Database, reformated data from the National Uranium Resource Evaluation (NURE) Hydrogeochemical and Stream Sediment Reconnaissance (HSSR) program: U. S. Geological Survey Open-File Report 97-492, version 1.20 (http://greenwood.cr.usgs.gov/pub/open-filereports/ofr-97-0492/).

Streufert, R.K., and Davis, M.W., 1990, Gold districts and placers of Colorado: Plate I, Colorado Geological Survey Resource Series 28, scale 1:500,000.

Walton-Day, K., Rossi, F.J., Gerner, L.J., Evans, J.B., Yager, T.J., Ranville, J.F., and Smith, K.S., 1999, Effects of fluvial tailings deposits on soils and surface- and ground-water quality, and implications for remediation-upper Arkansas River, Colorado, 1992-1996: U.S. Water Resources Investigations Report 99$4273,100 \mathrm{p}$. 